\title{
Indications to Promote Physical Activity during Pregnancy
}

\author{
Laura Stefani * (1), Gabriele Mascherini (D) and Giorgio Galanti \\ Sports Medicine Center, Clinical and Experimental Department, University of Florence-Italy, 50100 Florence, \\ Italy; gabriele.mascherini@unifi.it (G.M.); giorgio.galanti@unifi.it (G.G.) \\ * Correspondence: laura.stefani@unifi.it; Tel.: +39-34-7768-9030
}

Received: 3 July 2017; Accepted: 14 August 2017; Published: 14 August 2017

\begin{abstract}
Reduced physical activity in pregnancy is often associated with a progressive increase of chronic metabolic disease and to an enhanced risk for the child. The majority of women are less physically active during pregnancy, despite the motivation to improve their health, quality of life, and fitness. Education on the benefits of regular physical activity in pregnancy is determinant as suggested by obstetricians and gynecologists. The specific programs need to be supported by specialists in Sports Medicine. Counseling and support by nurses can also be helpful in encouraging women to maintain a sufficient level of physical activity during pregnancy or to modify their lifestyle, adapting the single models and specific physical activity programs to their needs.
\end{abstract}

Keywords: physical activity; health; pregnancy; lifestyle; exercise as prescription

\section{Introduction}

Pregnancy is a physiological condition for women during which the percentage of physical activity (PA) decreases. In this period, lifestyle generally changes and often tends to modify over time [1]. The progressive accumulation of chronic metabolic diseases is therefore commonly inevitable. The eventual onset of this situation is related to maternal and fetal risks. Literature reports how PA has been a topic of considerable research and attention in pregnancy [2], and evidence of the importance of PA for health promotion is highlighted. An appropriate level of PA or an exercise program during pregnancy is highly desirable. The choice to continue and to maintain health is a recent field of research in the area of maternal and child health [3], and pregnant women can be engaged in different levels of PA on the basis of their clinical conditions. The present brief report is intended to summarize the principal voices of the literature in order to verify any eventual discrepancies among the clinical indications supporting PA in pregnancy, particularly in the case of contraindications. A structured program with specific professional team figures such as sports medicine specialists and clinical exercise physiologists has recently become popular, and its application is increasing. The competence of these professional figures in this specific field and the potentially wide application of the program merit being divulged.

\section{Evidence of the Literature in Supporting Physical Activity and Exercise in Pregnancy}

This brief investigation was conducted by typing the principal key words such as pregnancy, physical activity, women's health, pregnancy weight gain, birth weight, obesity prevention, and nutritional intervention into PubMed, Google Scholar, Scopus, and Web of Science. The search included papers written between 2000 and 2017. Reviews and original articles addressing PA and exercise in pregnancy were considered. The results obtained showed evidence of the attention to PA in pregnancy. It emerged that women need to be motivated to change behavior-for example, by quitting smoking and 
improving nutrition. Pregnancy can be an opportunity for new patterns of behavior to be established to improve both maternal and fetal health.

A moderate level of PA is recommended by The American College of Obstetricians and Gynecologists and by the United States Department of Health and Human Services (ACOG/DHHS) [4]. In addition, the American College of Sports Medicine (ACSM) recommends pregnant women engage in at least 150 min of moderate-intensity physical activity weekly throughout pregnancy [5]. A normal level of PA is recommended during the postpartum period.

Most of the studies focused on aerobic exercise at moderate intensity (range 3-6 metabolic equivalents, METs) [6]. Fast walking, cycling, and some other leisure activities can reach this intensity [7]. In clinical practice, the amount of PA is easily established by using the 6-min walk test performed in association with the CR10 effort scale to estimate maximum perceived effort. This model for personalizing the amount of exercise prescribed has been proposed recently and tested in other clinical conditions such as cancer or chronic metabolic diseases [8]. Heart rate at the end of the test can be accepted as an adequate level of moderate intensity effort. Current guidelines define the length of time for the exercise proposed in greater detail.

A brief 10-to-15 min warm-up at lower intensity before aerobic exercise and a similar cool-down period at the end of the targeted aerobic activity is often suggested [5].

Regarding aerobic exercise, 35-90 min three-to-four times per week during pregnancy is considered safe and suggested for normal-weight women [9].

PA contributes to weight management in pregnancy and helps maintain body mass index (BMI) value at low risk level for the specific condition of pregnancy [10]. Aerobic exercise in overweight and obese pregnant women has been demonstrated to be associated with a significant prevention of gestational diabetes and a potential decrease in the rates of mellitus pre-eclampsia [11].

Therefore, in overweight and obese pregnant women, aerobic exercise for about 30-60 min three-to-seven times per week is suggested and PA should be encouraged [12]. PA also appears to be indicated for reducing the risk of premature birth. Particularly in antenatal intervention, a study recommends adequate lifestyles to reduce excess weight [13].

Few authors have investigated the safety and efficacy of strength training during pregnancy $[14,15]$. The Canadian guidelines suggest using lighter weights and more repetition, not lifting while lying flat on one's back, and avoiding the Valsalva maneuver [16]. However, considering the potential health-related benefits of strength training during pregnancy, further studies are required to identify the best model of exercise prescription in terms of volume and intensity.

In conclusion, the current literature can be divided into two different groups, according to the ACSM recommendations and the ACOG:

1. Pregnant women who can be encouraged to practice PA for their own well-being and that of their child [17];

2. Physically active women who can continue or increase their PA [18].

The real problem is that some women-especially those from social contexts with a low income and for whom the idea of practicing PA during pregnancy is not widely shared-contribute to maintaining physical activity at a low level. A definition of a dedicated program for an "in and out of hospital" context is relevant.

\section{Physical Activity: Definition and Implementations in Pregnancy}

PA is defined as any bodily movement produced by skeletal muscles that requires energy expenditure [19]. It is important to clarify that the PA model has different implications with respect to sports activity where competition, performance, and the optimization of athletic movement are the main characteristics.

The PA concept generally includes leisure, occupational, and domestic activities [20], as well as structured PA at major intensity. Despite the distinction of low intensity with respect to moderate-level 
PA, it is important to prepare a complete exercise program with the basic amount of PA necessary for reaching fitness level before starting with a specific program. This is essential, especially in pregnancy.

All daily basic PA produces energy expenditure. It is normally expressed in METs (metabolic equivalents) and is generally investigated on the basis of a simple questionnaire that refers to the well-known scale of correspondence between PA and energy expenditure [21]. The intensity of spontaneous daily PA is restricted in the range $<2$ METs. On the contrary, structured exercise corresponds to additional energy expenditure for each person. It is expressed in kcal per time unit and includes some elements in which all the structured physical movements are conceived to improve and maintain training which is fundamental.

A correct investigation of the two types of PA is the first step in planning a personalized model-especially for pregnant women. The current international guidelines do not completely adhere to this, particularly regarding the estimate of spontaneous daily PA that is often forgotten or neglected in the first analysis.

There is in fact some evidence as well as widespread general indications, supported, for example, by Canadian and American recommendations, that highlight the thesis that all pregnant women should engaged in PA throughout their pregnancy $[22,23]$. In the absence of critical clinical conditions such as the recurrence of bleeding, anemia, severe hypertension, rupture of membranes or evidence of cardiovascular disease, pregnant women should begin or maintain adequate PA throughout pregnancy [24] and continue it during the post-partum phase. Some general recommendations also include the importance of maintaining sufficient hydration, avoiding becoming overheated, and exercising in erect or supine positions after the first trimester, in order to contrast supine hypotension [25]. Some studies have also demonstrated that pregnant women have less nausea, fatigue, stress, and obesity [26] when following a prescribed exercise program.

Therefore, a personalized program with specific exercises appears to be important. However, from our research into the recent literature, no specific data on dedicated programs, with details of the competence of single professional figures involved, is available.

A model could be proposed, created by a first approach consisting of an interview regarding the lifestyle and habits of the pregnant women. In this phase, counseling by nursing staff would be valuable in order to verify the women's basic level of PA prior to pregnancy. Following this, the specific PA program should include consultation with the physician to rule out any eventual co-morbidities potentially limiting adherence to the program. The women should also undergo non-invasive evaluation of myocardial function by echocardiographic examination and Electrocardiographic exam (ECG).

As reported in the literature, in order to personalize the rate, intensity, and time of the aerobic exercises to be prescribed, the 6-min walk test is ideal. This is a simple test which is widely recognized to give sufficient information on the behavior of blood pressure and heart rate, corresponding to the moderate level of PA and effort tolerance. These data are essential for exercise prescription.

Nutritional counseling to investigate the quantity, quality, and daily distribution of food intake could be proposed for all pregnant women and not exclusively for obese or overweight women.

In our opinion, these are the basic parameters for sports medicine specialists planning exercise programs for pregnant women.

\section{To Engage and Support Pregnant Women in Physical Activity in Different Conditions}

The first step is to identify those subjects for whom following a program for the correct application of PA in pregnancy could be suggested. It is important to clarify that some common misconceptions about the amount and type of PA associated with improved perinatal outcomes are diffuse. There is some evidence that supports the positive role of PA in pregnancy. However, other evidence highlights the negative aspects due to bad positions that suggest a potential negative impact on breast milk production or infant growth [27]. A specific and personalized program needs to be proposed for women with uncomplicated pregnancies, taking the stage of pregnancy into consideration. The definition of 
"moderate-intensity physical activity" is in fact related to effort sufficient to raise heart and respiratory rates, while the ability to speak in short sentences remains [28].

Highly trained women often continue to exercise during pregnancy, but there is scarce specific information available to guide them. Evidence focusing on those who perform regular vigorous exercise suggests that this activity is helpful in preventing several symptoms in pregnancy, with little to no evidence of harm [29]. In women with gestational diabetes, particularly overweight and obese women, vigorous intense exercise during pregnancy has been seen to reduce excessive gestational weight gain [30]. However, transient fetal heart rate decelerations as well as altered uterine blood flow immediately after strenuous exercise - which raises concerns of reduced blood flow to the uterus-have been described [18]. It is therefore necessary to evaluate the fitness level of pregnant women before they engage in an exercise program [31]. During training, it is important for them to avoid exercising under the following contraindications:

1. Supine position after the first trimester

2. Prolonged standing position

3. High risk of contact PA, falling, or abdominal risk trauma

4. Altitudes over 5250 feet [32]

5. Scuba diving

6. Vaginal bleeding

7. Dizziness

8. Calf pain or swelling

9. Chest pain

10. Decreased fetal movement

11. Leakage of amniotic fluid

12. Dyspnea prior to physical activity

Although PA in pregnancy may be intuitive, few women are regularly engaged in a planned and personalized program, with the associated effective decrease in weight gain, risk of macrosomia, and general maternal and fetal cardiovascular risk. Only tailored exercise achieves the exercise goals.

These conditions do not exclude the potentially positive role of a specific PA session with indications for reducing back pain. In pregnancy, the locomotor system tends to adapt progressively to new biomechanical situations as a consequence of the increase in body weight that contributes to changing the musculoskeletal constraints of the column. The pelvis tilt is in anteversion with associated moving forward of the center of the abdomen, the lumbar section is positioned as lumbar hyperlordosis. Posture and balance stimulating proprioception is fundamental for reducing back pain. See comment in PubMed Commons below [33].

Finally, nutritional aspects cannot be separated from PA indications for pregnancy. There is a strong relation between PA and nutrition in terms of weight gain, but also with eventual nutritional imbalance. Particularly the latter has to be considered, in terms of relative contraindications that can induce severe anemia or extreme weight loss. Some general suggestions regarding nutritional habits are available for preventing the onset of chronic metabolic diseases such as diabetes. When a pregnant woman practices PA her diet needs to be increased by at least $300 \mathrm{kcal} /$ day mean value. In this context a specific professional figure is fundamental for planning her PA program.

\section{Discussion and Conclusions}

The first approach is to identify prejudice and unmask erroneous opinions in this field. Consultation by an obstetrician/gynecologist can increase the possibility of selecting subjects who can follow this program and share the culture of PA in pregnancy [34]. Counselling by nurses represents a first step, after which specialist medical expertise can personalize the indications. The role of nurses is useful in identifying pregnant women able to adhere to the PA program as well as in preventing 
the decrease of PA in pregnant women [35]. The specific competence of the sports medicine specialist, the physician, the clinical exercise physiologist, and the nutritionist can be helpful in setting the appropriate intensity of exercise.

A periodical follow-up for reassessing the level of physical activity appears to be fundamental [36]. In the majority of cases, the physiotherapist is involved in the modifications of the PA program in terms of intensity of the exercise model and in order to prevent or treat any low back pain [37] that occurs due to the progressive increase in weight throughout pregnancy.

However, the role of other professional figures such as the clinical exercise physiologist-dedicated to physical training - could be proposed for implementing the level of PA with respect to the stage of pregnancy. There is no evidence of this specific subject in the literature.

Nutritional counseling aimed at correcting unhealthy eating habits can be suggested. In addition, dietitians can also be helpful in prescribing a diet or suggesting alternative life-styles and habits [7]. Physicians specialized in sports medicine are actually considered the major experts in planning individually prescribed exercises for moderate intensity programs. However, insufficient data on this subject are reported in literature-especially in the context of sports medicine.

In recent literature, a new culture towards promoting PA in pregnancy appears to be emerging. Effective specialist team-work, reducing women's prejudices, and promoting PA has not yet been structured. Intervention is necessary throughout pregnancy as well as during the postpartum period. Diffusion of the results of research by various professional figures into the physiology of PA in pregnancy is called for.

Although the healthcare team involved in PA intervention for pregnant women is composed of various specialists, it is vital for researchers to continue to work and study in the field of physical activity. Their contributions will be determinant for optimizing programs for the future, and will support the primary and secondary prevention of chronic metabolic diseases among pregnant women.

Acknowledgments: No sources of funding have been used to support the study. We thank Susan Seeley for her English revision.

Author Contributions: Laura Stefani conceived, wrote and coordinated the manuscript. Gabriele Mascherini contributed to update the literature. Giorgio Galanti approved the final version of the paper.

Conflicts of Interest: The authors declare no conflict of interest.

\section{References}

1. Currie, S.; Sinclair, M.; Murphy, M.H.; Madden, E.; Dunwoody, L.; Liddle, D. Reducing the decline in physical activity during pregnancy: A systematic review of behaviour change interventions. PLoS ONE 2013, 8, e66385. [CrossRef] [PubMed]

2. Ette, L. Community-based exercise interventions during pregnancy are perceived as a satisfactory and motivating form of exercise engagement. Evid. Based Nurs. 2017, 20, 77-78. [CrossRef] [PubMed]

3. Campbell, F.; Johnson, M.; Messina, J.; Guillaume, L.; Goyder, E. Behavioral interventions for weight management in pregnancy: A systematic review of quantitative and qualitative data. BMC Public Health 2011, 11, 491. [CrossRef] [PubMed]

4. American Congress of Obstetrician Gynecologists. Physical activity and exercise during pregnancy and the postpartum period. Committee Opinion No. 650. Obstet. Gynecol. 2015, 126, e135-142.

5. American College of Sports Medicine; Pescatello, L.; Arena, R.; Riebe, D.; Thompson, P.D. Exercise prescription for healthy populations with special considerations and environmental considerations. In ACSM's Guidelines for Exercise Testing and Prescription; Baltimore, M.D., Ed.; Lippincott Williams \& Wilkins: Philadelphia, PA, USA, 2014; pp. 194-200.

6. Evenson, K.R.; Barakat, R.; Brown, W.J.; Dargent-Molina, P.; Haruna, M.; Mikkelsen, E.M.; Mottola, M.F.; Owe, K.M.; Rousham, E.K.; Yeo, S. Guidelines for physical activity during pregnancy: Comparisons from around the world. Am. J. Lifestyle Med. 2014, 8, 102-121. [CrossRef] [PubMed] 
7. Wang, C.; Wei, Y.; Zhang, X.; Zhang, Y.; Xu, Q.; Sun, Y.; Su, S.; Zhang, L.; Liu, C.; Feng, Y.; et al. A randomized clinical trial of exercise during pregnancy to prevent gestational diabetes mellitus and improve pregnancy outcome in overweight and obese pregnant women. Am. J. Obstet. Gynecol. 2017, 216, 340-351. [CrossRef] [PubMed]

8. Stefani, L.; Galanti, G.; Klika, R. Clinical Implementation of Exercise Guidelines for Cancer Patients: Adaptation of ACSM's Guidelines to the Italian Model. J. Funct. Morphol. Kinesiol. 2017, 2, 4. [CrossRef]

9. Di Mascio, D.; Magro-Malosso, E.R.; Saccone, G.; Marhefka, G.D.; Berghella, V. Exercise during pregnancy in normal-weight women and risk of preterm birth: A systematic review and meta-analysis of randomized controlled trials. Am. J. Obstet. Gynecol. 2016, 215, 561-571. [CrossRef] [PubMed]

10. Institute of Medicine (US) and National Research Council (US) Committee to Reexamine IOM Pregnancy Weight Guidelines; Rasmussen, K.M.; Yaktine, A.L. Weight Gain during Pregnancy: Reexamining the Guidelines; National Academies Press: Washington, DC, USA, 2009; pp. 1-4.

11. Mauman, A.; Mannan, M.; Doi, S. Gestational weight gain in relation to offspring obesity over the life course: A systematic review and bias-adjusted metaanalysis. Obes. Rev. 2014, 15, 338-347. [CrossRef] [PubMed]

12. Magro-Malosso, E.R.; Saccone, G.; di Mascio, D.; di Tommaso, M.; Berghella, V. Exercise during pregnancy and risk of preterm birth in overweight and obese women: A systematic review and meta-analysis of randomized controlled trials. Acta Obstet. Gynecol. Scand. 2017, 96, 263-273. [CrossRef] [PubMed]

13. Rankin, J. Effects of Antenatal Exercise on Psychological Well-Being, Pregnancy and Birth Outcome. J. Adv. Nurs. 2003, 41, 623.

14. O'Connor, P.J.; Poudevigne, M.S.; Cress, M.E.; Motl, R.W.; Clapp, J.F., III. Safety and efficacy of supervised strength training adopted in pregnancy. J. Phys. Act. Health 2011, 8, 309-320.

15. Barakat, R.; Lucia, A.; Ruiz, J.R. Resistance exercise training during pregnancy and newborn's birth size: A randomised controlled trial. Int. J. Obes. 2009, 33, 1048-1057. [CrossRef] [PubMed]

16. Wolfe, L.; Mottola, M. Physical Activity Readiness Medical Examination for Pregnancy: PARmed-X for Pregnancy; Canadian Society of Exercise Physiology \& Health Canada: Ottawa, ON, Canada, 2002.

17. Petersen, A.M.; Leet, T.L.; Brownson, R.C. Correlates of physical activity among pregnant women in the United States. Med. Sci. Sports Exerc. 2005, 37, 1748-1753. [CrossRef] [PubMed]

18. Salvensen, K.A.; Hem, E.; Sundgot-Borgen, J. Fetal wellbeing may be compromised during strenuous exercise among pregnant elite athletes. Br. J. Sports Med. 2012, 46, 279-283. [CrossRef] [PubMed]

19. World Health Organization (WHO). Global Recommendations on Physical Activity for Health; World Health Organization: Geneva, Switzerland, 2010.

20. Caspersen, C.J.; Powell, K.E.; Christenson, G.M. Physical activity, exercise, and physical fitness: Definitions and distinctions for health-related research. Public Health Rep. 1985, 100, 126-131. [PubMed]

21. McArdle, W.; Katch, F.I.; Katch, V.L. Exercise Physiology: Nutrition, Energy, and Human Performance, 8th ed.; Wolter Kluwer, Health: Alphen aan den Rijn, The Netherlands, 2014.

22. ACOG Committee Obstetric Practice. ACOG Committee opinion. Number 267, January 2002: Exercise during pregnancy and the postpartum period. Int. J. Gynaecol. Obstet. 2002, 77, 79-81.

23. Artal, R.; O'Toole, M. Guidelines of the American College of Obstetricians and Gynecologists for exercise during pregnancy and the postpartum period. Br. J. Sports Med. 2003, 37, 6-12. [CrossRef] [PubMed]

24. Zavorsky, G.; Longo, L. Exercise guidelines in pregnancy. Sports Med. 2011, 41, 345-360.

25. Mottola, M.F. Physical activity and maternal obesity: Cardiovascular adaptations, exercise recommendations, and pregnancy outcomes. Nutr. Rev. 2013, 71, S31-S36. [CrossRef] [PubMed]

26. Clapp, J.F. Morphometric and neurodevelopmental outcome at age five years of the offspring of women who continued to exercise regularly throughout pregnancy. J. Pediatr. 1996, 129, 856-863. [CrossRef]

27. Polley, B.; Wing, R.; Sims, C. Randomized controlled trial to prevent excessive weight gain in pregnant women. Int. J. Obes. 2002, 26, 1494-1502. [CrossRef] [PubMed]

28. Rönö, K.; Stach-Lempinen, B.; Klemetti, M.M.; Kaaja, R.J.; Pöyhönen-Alho, M.; Eriksson, J.G.; Koivusalo, S.B. Prevention of gestational diabetes through lifestyle intervention: Study design and methods of a Finnish randomized controlled multicenter trial. BMC Pregnancy Childbirth 2014, 14, 70. [CrossRef] [PubMed]

29. Pivarnik, J.M.; Szymanski, L.M.; Conway, M.R. The Elite Athlete and Strenuous Exercise in Pregnancy. Clin. Obstet. Gynecol. 2016, 59, 613-619. [CrossRef] [PubMed] 
30. Ehrlich, S.F.; Sternfeld, B.; Krefman, A.E.; Hedderson, M.M.; Brown, S.D.; Mevi, A.; Chasan-Taber, L.; Quesenberry, C.P., Jr.; Ferrara, A. Moderate and Vigorous Intensity Exercise during Pregnancy and Gestational Weight Gain in Women with Gestational Diabetes. Matern. Child Health J. 2016, 20, 1247-1257. [CrossRef] [PubMed]

31. Hinman, S.K.; Smith, K.B.; Quillen, D.M.; Smith, M.S. Exercise in Pregnancy: A Clinical Review. Sports Health 2015, 7, 527-531. [CrossRef] [PubMed]

32. Entin, P.L.; Coffin, L. Physiological Basis for Recommendations Regarding Exercise during Pregnancy at High Altitude. High Alt. Med. Biol. 2004, 5, 321-334. [CrossRef] [PubMed]

33. Abu, M.A.; Abdul Ghani, N.A.; Shan, L.P.; Sulaiman, A.S.; Omar, M.H.; Ariffin, M.H.M.; Baharuddin, A.; Shohaimi, S.; Man, Z.C. Do exercises improve back pain in pregnancy? Horm. Mol. Biol. Clin. Investig. 2017. [CrossRef] [PubMed]

34. Bredin, S.S.; Foulds, H.J.; Burr, J.F.; Charlesworth, S.A. Risk assessment for physical activity and exercise clearance: In pregnant women without contraindications. Can. Fam. Physician 2013, 59, 515-517. [PubMed]

35. Engberg, E.; Stach-Lempinen, B.; Rönö, K.; Kautiainen, H.; Eriksson, J.G.; Koivusalo, S.B. A randomized lifestyle intervention preventing gestational diabetes: Effects on self-rated health from pregnancy to postpartum. J. Psychosom. Obstet. Gynaecol. 2017, 9, 1-6. [CrossRef] [PubMed]

36. Tinius, R.A.; Cahill, A.G.; Cade, W.T. Origins in the Womb: Potential Role of the Physical Therapist in Modulating the Deleterious Effects of Obesity on Maternal and Offspring Health through Movement Promotion and Prescription during Pregnancy. Phys. Ther. 2016. [CrossRef] [PubMed]

37. Bishop, A.; Holden, M.A.; Ogollah, R.O.; Foster, N.E.; EASE Back Study Team. Current management of pregnancy-related low back pain: A national cross-sectional survey of U.K. physiotherapists. Physiotherapy 2016, 102, 78-85. [CrossRef] [PubMed]

(C) 2017 by the authors. Licensee MDPI, Basel, Switzerland. This article is an open access article distributed under the terms and conditions of the Creative Commons Attribution (CC BY) license (http://creativecommons.org/licenses/by/4.0/). 